\title{
CONSUMO DE ÁlCOOL EM ADOLESCENTES DE UMA ESCOLA DA REDE PUBLICA DE ENSINO DO MUNICIPIO DE PONTA GROSSA (PR)
}

Thiago Krzesinski dos Santos

Fabricio Cieslak

Suelen Meira Goes

Alex Pinheiro Gordia

Teresa Maria Bianchini de Quadros

Gustavo Levandoski

Guanis de Barros Vilela Junior

Neiva Leite

\section{Resumo}

Analisar o alcoolismo em adolescentes de uma escola da rede publica de ensino. Participaram do estudo 63 estudantes de escola da rede publica de ensino do município de Ponta Grossa (PR) (39 do sexo feminino e 24 do sexo masculino). O consumo de álcool foi mensurado pelo AUDIT e a consistência interna atraves do coeficiente Alfa de Cronbach. Para analise do consumo de álcool recorreu-se a estatística descritiva e o teste de Qui-Quadrado para verificar as possíveis associates do álcool ao sexo. Os resultados demonstram que o consumo de álcool foi de baixo risco e não foram encontradas associates entre o alcoolismo e o sexo para esta pesquisa.

\section{Palavras-Chave}

Adolescentes; Alcoolismo; AUDIT.

\section{ALCOHOL CONSUMPTION IN ADOLESCENTS OF A SCHOOL OF THE NET PUBLISHES OF EDUCATION OF PONTA GROSSA CITY (PR)}

Thiago Krzesinski dos Santos

Fabrício Cieslak

Suelen Meira Goes

Alex Pinheiro Gordia

Teresa Maria Bianchini de Quadros

Gustavo Levandoski

Guanis de Barros Vilela Junior

Neiva Leite

\begin{abstract}
Analyzing the alcoholism in teenagers of a school in the public school system. Part of the study 63 students from school in the public school system of the city of Ponta Grossa (PR) (39 female and 24 male). The consumption of alcohol was measured by the AUDIT and the internal consistency by the Cronbach alpha coefficient. For analysis of alcohol consumption appealed to descriptive statistics and chi-square test to verify the possible associations of alcohol to sex. The results showed that alcohol consumption was low risk and were not found associations between alcohol and sex for this search.
\end{abstract}

\section{Key-Words}

Teenagers; Alcoholism; AUDIT. 


\section{INTRODUÇÃO}

$\mathrm{Na}$ atualidade muitos estudos vem sendo realizados com o intuito de verificar o consumo de álcool em diversas populações (GORDIA; QUADROS; LEITE; KALINOWSKI, 2005; PICKARD; BATES; DORIAN; GREIG; SAINT, 2000), no sentido que os problemas relacionados ao uso do álcool sao evidentes e estão relacionados a $54 \%$ dos acidentes de trabalho com afastamento e $40 \%$ dos acidentes com morte (AMARAL; MALBIERGER, 2004).

Evidencias tem indicado que o álcool e a substancia psicoativa mais consumida entre os jovens (KERR-CORREA, ANDRADE, BASSIT, BOCCUTO, 1999; STEMPLLUK, 2005), fato preocupante na saúde pública, de modo que, o alcoolismo esta associado a diversos problemas como: mortes no transito, desentendimentos familiares e afetivos, homicídios, espancamentos, deserção do trabalho e da escola (COSTA DO NASCIMENTO; JUSTO, 2000). Acredita-se que o prévio conhecimento de fatores associados ao uso de drogas em jovens no pais e de grande relevância, de modo a permitir possíveis intervenções sobre comportamentos e fatores de risco com o intuito de inibir o consumo abusivo e progressivo, vicio deletério para o jovem, de drogas licitas e ilícitas (SOLDERA; DALGALARRONDO; CORREA FILHO, SILVA, 2004).

O diagnostico do alcoolismo e embasado em quatro grupos de indicadores: consumo, dependência psicológica, dependência física e problemas relacionados ao álcool (SCHUCKIT, 1985). Segundo a Organização Mundial da Saúde (OMS), as taxas limitrofes para baixo risco do consumo de bebidas álcoolicas para as mulheres sao de ate duas doses e para os homens de ate três doses (BABOR, HIGGINS-BIDDLE; SAUNDERS; MONTEIRO, 2001).

A prevalência do alcoolismo identifica uma quantia substancial dos pacientes atendidos na emergência, avalia-se nos Estados Unidos que 110 milhões de visitas a estes serviços estão diretamente relacionadas ao consumo de álcool (D'ONOFRIO; DEGUTIS, 2002). Para a OMS o uso indevido de álcool impõe as sociedades a uma quantidade considerável de agravos indesejáveis, de forma que, o consumo de bebidas alcoólicas corresponde a 1,5\% de mortes e 3,5\% de DALYS (Disability-Adjusted Life Years) (WHO, 2001).

Em estudantes as Conseqüências se estendem ao baixo desempenho relacionado as faltas e ao rendimento nas aulas e exames das disciplinas, muitas vezes devido ao fato de os alunos freqüentarem festas noturnas e ficarem intoxicados (binge drinking) (MCGEE; KYPRI, 2004). Alem disso, o 
alcoolismo dentre os jovens podem acarretar outras Conseqüências como: sexo desprotegido, acidentes automobilísticos e envolvimento em brigas e atitudes de violência (PECHANSKY; SZOBOT, SCIVOLLETO, 2004).

Baseando-se nessas informações, o presente estudo objetivou analisar o consumo de bebidas alcoólicas em adolescentes de uma escola de rede publica de ensino.

\section{PROCEDIMENTOS METODOLÓGICOS}

O estudo envolveu um delineamento observacional, descritivo e transversal, sendo realizado no município de Ponta Grossa (PR), no ano de 2007. Participaram do estudo 63 estudantes (39 do sexo feminino e 24 do sexo masculino) de uma escola de rede publica de ensino do referido município. Apos detalhado esclarecimento sobre os propósitos dessa investigação, procedimentos utilizados, benefícios e possíveis riscos atrelados, os participantes assinaram termo de consentimento, condicionando sua participação de modo voluntário.

A mensuração do consumo de álcool foi realizada pelo Alcohol Use Disorders Identification Test (AUDIT), instrumento elaborado pela OMS (BABOR; LA FUENTE; SAUNDERS, GRANT, 1992) contendo 10 questões (três relacionadas ao uso de álcool, quatro sobre dependência e três sobre problemas decorrentes do consumo) com respostas fechadas de múltipla escolha e abordando o padrão de consumo de bebidas a suas Conseqüências nos últimos 12 meses. O escore varia de 0 a 40 pontos e sua classificação baseia-se em quatro níveis conforme o consumo etílico: "consumo de baixo risco ou abstemios" $=0$ a 7 pontos, "consumo de risco" $=8$ a 15 pontos, "Consumo de alto risco ou uso nocivo" = 16 a 19 pontos e "provável dependência" $=20$ ou mais pontos (maximo = 40 pontos).

Segundo a literatura, este questionário apresentou validação com uma sensibilidade de $92 \%$ e especificidade de 3\% (BABOR, HIGGINS-BIDDLE, SAUNDERS, MONTEIRO, 2001). O AUDIT foi traduzido (FIGLIE; PILLON; LARANJEIRA; DUNN; 1997) e obteve sua validação na versão brasileira (LIMA; FREIRE; SILVA; TEIXEIRA; FARREL; PRINCE; 2005).

Para a analise dos resultados foram utilizadas medidas descritivas para verificar o consumo de álcool dos indivíduos, em seguida empregou-se o teste de Qui-Quadrado com o intuito de localizar as possíveis associates do consumo etílico ao sexo, o nível adotado foi de significância de $p<0,05$. A verificação da consistência interna das respostas do instrumento foi obtida por intermédio do 
coeficiente de fidedignidade Alfa de Cronbach (a). O tratamento estatístico foi realizado mediante a utilização do software Statistical Package for the Social Sciences (SPSS, versão 13.0) for Windows.

\section{RESULTADOS E DISCUSSÃO}

Os resultados obtidos na analise da consistência interna das respostas, mediante a utilização do coeficiente de fidedignidade de Alfa Cronbach para todos os grupos sao apresentados na Tabela 1.

Tabela 1 - Valores dos coeficientes de fidedignidade obtidos atraves das respostas do questionário AUDIT.

\begin{tabular}{ccl}
\hline $\begin{array}{c}\text { Consumo de } \\
\text { Álcool } \\
\text { Coeficiente }\end{array}$ & $\begin{array}{c}\text { Feminino } \\
\text { Ponta Grossa }\end{array}$ & $\begin{array}{l}\text { Masculino } \\
\text { Ponta Grossa }\end{array}$ \\
Cronbach (a) & 0,89 & 0,79 \\
\hline
\end{tabular}

Os valores da consistência interna das respostas obtidos atraves do coeficiente de fidedignidade de Alfa Cronbach (a) foram satisfatórios para todos os grupos de uma forma geral. Conforme Thompson (1999), o importante e compreender a confiabilidade estimada como uma função dos resultados obtidos em um instrumento, ou seja, os valores dos coeficientes resultantes revelam a confiabilidade das respostas obtidas nesta pesquisa.

Foram obtidos atraves da aplicação do instrumento AUDIT os resultados referentes ao consumo de álcool, divididos em quatro níveis de escore da seguinte forma: Consumo de Baixo Risco ou Abstinência, Consumo de Risco, Consumo de Alto Risco ou Uso Nocivo e Provável Dependência, sao apresentados na Tabela 2.

Tabela 2 - Valores do AUDIT apresentados por níveis de escore.

\begin{tabular}{ccc}
\hline $\begin{array}{c}\text { Consumo de } \\
\text { Alcool }\end{array}$ & $\begin{array}{c}\text { Feminino } \\
\text { Ponta Grossa }\end{array}$ & $\begin{array}{c}\text { Masculino } \\
\text { Ponta Grossa }\end{array}$ \\
\hline $\begin{array}{c}\text { Consumo de } \\
\text { Baixo Risco } \\
\text { Consumo de } \\
\quad \text { Risco } \\
\text { Consumo de } \\
\text { Alto Risco } \\
\text { Provável } \\
\text { Dependência }\end{array}$ & $77 \%(30)$ & $83,3 \%(20)$ \\
\hline$* \mathrm{p}<0,05$ & $10,2 \%(4)$ & $12,5 \%(3)$ \\
& $12,8 \%(5)$ & $0 \%(0)$ \\
& & $4,2 \%(1)$ \\
\hline
\end{tabular}


Os resultados demonstram que as maiorias dos adolescentes apresentam um consumo de baixo risco tanto para o sexo feminino $(77 \%)$ como no masculino $(83,3 \%)$. Em outra pesquisa, desenvolvida por Gordia, Quadros, Leite e Kalinowski (2005), com 193 adolescentes do ensino médio da área rural do município da Lapa (PR), identificou-se que 69,9\% dos indivíduos foram classificados como consumidores de baixo risco e 30,1\% estiveram nas classes de consumo de risco e/ou consumo de alto risco e/ou provável dependência.

Foi verificado que $12,8 \%$ das adolescentes do sexo feminino e 4,2\% dos indivíduos do sexo masculino apresentaram-se como prováveis dependentes de bebidas alcoólicas. Em estudo realizado com universitários da Nova Zelândia por Kypri, Langley, Mcgee, Saunders e Williams (2002), os resultados indicaram que $60 \%$ dos homens e 58,2\% das mulheres consomem bebidas alcoólicas acima dos níveis recomendados pela OMS.

O teste Qui-Quadrado $\left({ }^{\wedge}=0,827\right)$ não identificou associações significativas $(p=0,363)$ em relação ao consumo etilico e o sexo. Evidencias similares foram encontradas na pesquisa de Souza, Areco e Silveira Filho (2005), que estudaram 2718 adolescentes da rede estadual de ensino do município de Cuiabá (MT) e examinaram que o consumo de álcool não esta associado ao gênero. Entretanto, outras pesquisas mostraram que os homens consomem mais álcool em relação as mulheres (ALVES; COSTA; SOBRINHO; SANTOS; GOMES; ASSIS; 2005).

\section{CONSIDERAÇÕES FINAIS}

Com base nos resultados o presente estudo possibilitou uma visão reflexiva sobre o alcoolismo, podendose concluir que o consumo de álcool dos adolescentes foi de baixo risco e que o mesmo não esteve associado ao sexo.

\section{REFERÊNCIAS}

ALVES, M.V. Q. M.; et al. Uso de bebidas alcoólicas entre adolescentes: perfil de experimentação, uso regular e fatores de risco. Revista Baiana de Saúde Publica, Salvador, v. 29, n. 1., p.95-100, 2005. AMARAL, R. A.; MALBIERGER, A. A avaliação de instrumento de detecção de problemas relacionados ao uso do álcool (CAGE) entre trabalhadores da Prefeitura do campus da Universidade de Sao Paulo (USP) - Campus capital. Revista Brasileira de Psiquiatria, Sao Paulo, v. 26, n. 3., p. 156163, set. 2004. 
KYPRI, K.; LANGLEY, J. D.; MCGEE, R.; SAUNDERS, J. B.; WILLIAMS, S. High prevalence, persistent hazardous drinking among New Zealand tertiary students. Alcohol \& Alcoholism, Oxford, v. 37, n. 5, p. 457-464, sep. 2002.

LIMA, C.T.; et al. Concurrent and construct validity of the AUDIT in the urban Brazilian sample. Alcohol \& Alcoholism, Oxford, v. 40, n. 6, p.584-589, sep. 2005.

MCGEE, R.; KYPRI, K. Alcohol-related problems experienced univesity students in New Zealand. Australian and New Zealand Joumal Public Health, Melboume, v.28, n.4., p.321-323, aug. 2004

PECHANSKY, F.; SZOBOT, C. M.; SCIVOLlETO, S. Uso de álcool entre adolescentes: conceitos, características epidemiológicas e fatores etiopatogenicos. Revista Brasileira de Psiquiatria, Sao Paulo, v. 6, supl. 1., p.14-17, mai. 2004

PICKARD, M.; et al. Alcohol and drug use in second-year medical students at the University of Leeds. Medical Education, Oxford, v.34, n.3., p.148-150, feb. 2000

SCHUCKIT, M.A. Alcohol pattems and problems. New Jersey: Rutgers University Press, 1985.

SOLDERA, M.; et al. Uso de drogas psicotropicas por estudantes: prevalência e fatores associados. Revista de Saúde Publica, Sao Paulo, v. 38, n. 2, p. 277-283, abr. 2004.

SOUZA, D. P. O.; ARECO, K. N.; SILVEIRA FILHO, D.X. Álcool e alcoolismo entre adolescentes da rede estadual de ensino de Cuiabá, Mato Grosso. Revista de Saúde Publica, Sao Paulo, v. 39, n. 4, p.585-592, ago. 2005.

STEMPllUK, V. A. Uso de drogas entre alunos da Universidade de Sao Paulo: 1996 versus 2001. Revista Brasileira de Psiquiatria, Sao Paulo, v. 27, n. 3, p. 185-193, abr/jun. 2005.

THOMPSON, B. Score reliability. Texas: Texas A \& M University, 1999.

WORLD HEALTH ORGANIZATION. The World Health Report 2001: mental health: new understanding, new hope. Geneva: 2001. 


\section{Thiago Krzesinski dos Santos}

Universidade Federal do Parana

\section{Fabricio Cieslak \\ Universidade Federal do Parana}

\section{Suelen Meira Goes}

Universidade Federal do Parana

\section{Alex Pinheiro Gordia \\ Universidade Federal do Parana}

\section{Teresa Maria Bianchini de Quadros}

Universidade Federal de Santa Catarina

\section{Gustavo Levandoski}

Universidade do Estado de Santa Catarina

\section{Guanis de Barros Vilela Junior}

Universidade Estadual de Ponta Grossa

\section{Neiva Leite \\ Universidade Federal do Parana}

\section{Referência do artigo:}

\section{ABNT}

SANTOS, T. K. et. al. Consumo de álcool em adolescentes de uma escola da rede pública de ensino do município de Ponta Grossa (PR). Conexões, v. 6, p. 254-260, 2008.

\section{APA}

Santos, T. K., Cieslak, F., Goes S. M., Gordia, A. P., Quadros, T. M. B., Levandoski, G., Junior, G. B. V., \& Leite, N. (2008). Consumo de álcool em adolescentes de uma escola da rede pública de ensino do município de Ponta Grossa (PR). Conexões, 6, 254-260.

\section{VANCOUVER}

Santos TK, Cieslak F, Goes SM, Gordia AP, Quadros TMB, Levandoski G, Junior GBV, Leite N. Consumo de álcool em adolescentes de uma escola da rede pública de ensino do município de Ponta Grossa (PR). Conexões, 2008; 6: 254-260. 